\title{
LA LECTURA LITERARIA AL EGRESO DE LA FORMACIÓN INICIAL DOCENTE. UN RETRATO DE LAS PRÁCTICAS LECTORAS DECLARADAS POR ESTUDIANTES DE PEDAGOGÍA EN EDUCACIÓN SECUNDARIA EN LENGUAJE Y COMUNICACIÓN
}

\author{
Eduardo Asfura ${ }^{1}$, \\ Neus Real ${ }^{2}$
}

RESUMEN

El estudio presenta un retrato de las prácticas de lectura literaria declaradas por estudiantes de Pedagogía en educación secundaria en Lenguaje y Comunicación de cuatro universidades de Santiago de Chile, al momento del egreso de la formación inicial docente. Desde la opción metodológica de los estudios sincrónicos y estadísticos, la investigación entrega una representación descriptiva del entorno familiar y social para la lectura, el lugar de la actividad lectora en el uso del tiempo libre y las prácticas de lectura literaria. En el retrato se distingue entre las prácticas lectoras asociadas al cumplimiento académico y las lecturas libres, considerando variables como la frecuencia, cantidad, preferencias, formas de acceso, autores y obras. En sus resultados y conclusiones, el estudio revela un hábito lector en formación, antes que una relación significativa con la literatura. La poca influencia del entorno familiar en los hábitos de lectura, la escasa diversidad de géneros frecuentados, así como la resistencia a las formas más experimentales de la producción literaria, remiten a un encuentro más bien tardío con la experiencia literaria, determinado por una fuerte tensión entre la lectura personal y la que deriva de las exigencias de la formación académica.

Conceptos clave: didáctica de la literatura, formación inicial docente, lectura literaria, prácticas lectoras.

LITERARY READING PRACTICES OF RECENT TEACHER EDUCATIONGRADUATES. A PORTRAIT OF READING PRACTICES AS DECLARED BY FUTURE SECONDARY EDUCATION LANGUAGE ARTS TEACHERS

\section{ABSTRACT}

This study presents a portrait of self-declared literary reading practices by recent graduates of Secondary Education Language Arts teacher education programs from four universities, in Santiago de Chile. From the methodological option of synchronic and statistical studies, this research provides a descriptive representation of the family and social environment for

Universidad San Sebastián, Santiago, Chile. Contacto: easfurai@docente.uss.cl

2 Universidad Autónoma de Barcelona, Barcelona, España. Contacto: Neus.Real@uab.cat 
reading, where reading takes place in leisure time and literary reading practices. The portrait distinguishes between reading practices associated with fulfilling academic demands and leisure reading, considering variables such as frequency, quantity, preferences, access, authors and works. The study's results reveal an emerging reader habit, rather than a meaningful relationship with literature. The near absent influence of the family environment on reading habits, the scarce diversity of genres frequented, as well as the resistance to more experimental forms of literary production, are associated with a delayed encounter with the literary experience, which is determined by a strong tension between reading for pleasure or to meet the demands of academic training.

Key concepts: initial teacher training, literary reading, reading practices, teaching of literature.

\section{Introducción}

Aunque tradicionalmente se piensa al estudiante de Pedagogía Media en Lenguaje y Comunicación (PMLC, en adelante) como un buen lector de literatura, a la manera clásica o humanista de serlo, los cambios de las últimas tres décadas en el sistema de educación superior en Chile, así como las transformaciones de la cultura escrita en el conjunto de la sociedad global, han diversificado las prácticas y experiencias culturales de los universitarios chilenos. Tal como describen Jiménez y Lagos (2011), desde comienzos de la década de 1980 la educación superior chilena ha transitado desde un modelo homogéneo y altamente selectivo, integrado por un total de ocho universidades, a un nuevo sistema compuesto por 60 universidades, 44 institutos profesionales y 73 centros de formación técnica. Esta expansión, que ha posibilitado el acceso a las universidades e institutos de jóvenes no pertenecientes a la élite, ha estado no obstante caracterizada por una "continua desigualdad en las tasas de acceso a la universidad y retención, y una segmentación de clase según las universidades de destino de los estudiantes" (Leyton, Vásquez y Fuenzalida, 2012, p. 62).

La ampliación de la cobertura del sistema de educación superior y la diversificación de los perfiles de ingreso a la universidad suponen importantes desafíos y nuevos modos de relación con el conocimiento y la cultura en la formación universitaria, los que resultan particularmente sensibles en la formación del profesorado de PMLC y el rol que estos futuros pedagogos deberán asumir como mediadores escolares de lectura literaria. 
Las exigencias de dicho rol profesional se hallan claramente representadas en la noción de "sujeto lector didáctico" (Munita, 2018), que destaca la relación entre la identidad lectora de los profesores y su actuación docente. En este sentido, el interés por investigar las prácticas lectoras de los profesores se basa en el supuesto que "los profesores que son buenos lectores son, también, mejores mediadores en el aprendizaje lector y literario de los niños y jóvenes" (2018, p. 3). Aunque esta relación no es automática ni directa y debe valorarse a la luz de los factores contextuales del desempeño profesional, la evidencia científica demuestra una indudable influencia entre la vida lectora de los maestros y las posibilidades de su actuación didáctica en la formación de lectores ${ }^{3}$.

El conjunto de estos desafíos se enmarca en las actuales líneas de avance de la didáctica de la literatura, cuyas bases al respecto remiten a la propuesta de lectura transaccional de Rosenblatt (2002) las ideas acerca de recepción estética y poética del lector de Jauss (1992) y, en general, al enfoque pragmático-comunicativo del discurso. En el contexto de dichas propuestas, resulta claro que el tránsito desde la tradicional enseñanza de la literatura al desarrollo de la educación literaria exige competencias de mediación didáctica que no solo se forman desde los saberes conceptuales del currículo (Colomer, 2005). El desafío de "propender al logro de objetivos afectivos, cognitivos, metalingüísticos y ético-discursivos de los estudiantes" (Sánchez Corral, 2008, p. 327), recuperando los aspectos epistemológicos de la experiencia estético-literaria para la formación de la competencia literaria, hace predecir que su tratamiento didáctico exige no solo conocimientos disciplinarios sobre literatura, sino experiencias vitales de lectura literaria, que permitan al profesor disponer de los criterios para, por ejemplo, recomendar obras y valorar la recepción que sus estudiantes hagan de ellas, en un contexto en el que la figura del mediador escolar se tensiona ante "la deslocalización de las nuevas lecturas de la escuela"

Un ejemplo del papel que, en este sentido, están llamadas a cumplir las instituciones formadoras de profesores en Chile es la interesantísima iniciativa Biblioteca Lea, de la Universidad Diego Portales, creada especialmente para que el programa académico de las carreras de Pedagogía desarrolle competencias y habilidades de mediación lectora en los futuros profesores. 
(Lluch, 2010, p. 105) y el arribo de nuevas tecnologías, prácticas y circuitos de consumo cultural juveniles.

En el ámbito internacional, las investigaciones que vinculan las prácticas lectoras con la formación inicial docente, como las de Applegate y Applegate (2004), Gupta (2004), Pascual Díez (2007), Munita (2013) y Granado y Puig (2014), expresan una realidad que, aunque esperable, es escasamente considerada en el diseño e implementación de las propuestas de formación del profesorado: las prácticas lectoras de los futuros maestros y maestras han cambiado, tal como ha sucedido con la relación entre la palabra escrita y las prácticas lectoras del conjunto de la sociedad. Y dicha transformación compromete la cantidad y frecuencia de lecturas, pero también las motivaciones, formas de acceso y soportes que los sujetos escogen para leer. En este sentido, los itinerarios y prácticas lectoras de muchos de los nuevos profesores en formación parecen hallarse más cerca de las de sus futuros estudiantes de secundaria que de las de sus maestros universitarios, revelando al menos tres grandes fenómenos:

a. Un escaso y poco variado repertorio de lecturas literarias personales distintas de la lectura prescriptiva que la vida universitaria les impone como parte del cumplimiento de actividades académicas;

b. la concepción de la literatura como una "materia a aprender" (o enseñar), atribuyendo a los aspectos históricos del objeto literario un mayor valor que a la propia experiencia de recepción lectora y, estrechamente vinculado con ello;

c. una valoración casi estrictamente teórica y conceptual de las obras de literatura clásica y contemporánea que han "debido leer" en la escuela o la universidad, en contraste con el carácter más libre de sus impresiones acerca de los libros que leen por gusto y/o interés personal.

Aunque los hábitos y prácticas lectoras declaradas por los universitarios chilenos pueden suponerse representadas, de modo muy general, en las encuestas nacionales de lectura de los últimos 
años ${ }^{4}$, la bibliografía no reporta investigaciones que aborden en Chile las prácticas lectoras de estudiantes de PMLC. Los dos estudios que más se aproximan a esta población son el de Claudia Gilardoni (2006) y el de Juan Eduardo García-Huidobro (2009). En el primer caso se trata de una investigación que explora las prácticas lectoras de 834 estudiantes universitarios de pregrado de Ciencias y Humanidades de la Región Metropolitana. Entre sus interesantes conclusiones, Gilardoni reveló que un tercio de los participantes manifiesta no leer nunca, casi nunca u ocasionalmente, reportándose además una valoración de la lectura predominantemente referencial y utilitaria, más que estética y recreativa. La investigación de García-Huidobro, en tanto, encuestó a 80 profesores de la Facultad de Educación de la Universidad Alberto Hurtado, indagando en sus usos pedagógicos y concepciones acerca de la lectura en la formación de profesores. Entre sus resultados, la investigación reportó altas valoraciones y expectativas por parte de los docentes en torno a la importancia de la lectura para la formación de profesores, las que sin embargo contrastan con el riesgo de que el uso de los textos en el aula promueva "en los estudiantes el desarrollo de estrategias orientadas a leer para responder" (2009, p. 164).

A pesar de su relevancia, estas indagaciones acerca del comportamiento lector universitario no abordan las prácticas de lectura literaria de los futuros profesores de Lenguaje y Comunicación de educación secundaria, quienes tendrán la responsabilidad de mediar, de manera directa, en el desarrollo del hábito lector y la educación literaria de los jóvenes de enseñanza media del país. De este modo, explorar y caracterizar las prácticas de lectura literaria que declaran estudiantes de PMLC al momento de su egreso de la formación inicial docente se ha considerado un primer paso

\footnotetext{
Entre estas encuestas e investigaciones cabe destacar los estudios acerca del comportamiento lector del Consejo de la Cultura y las Artes de los años 2011 y 2014, elaborados por el Centro de Microdatos de la Universidad de Chile y la Dirección de Estudios Sociales de la Pontificia Universidad Católica de Chile, respectivamente; las tres investigaciones sobre los hábitos y preferencias de lectura, tenencia y compra de libros que, con el título de Chile y los libros, realizaron entre 2006 y 2010 la Fundación La Fuente y Adimark GFK. Junto con ellos, la investigación de Alberto Mayol, La lectura como práctica social: condiciones de fortalecimiento y debilitamiento de la lectura en Chile (2014), en el Observatorio del Libro y la Lectura, aporta interesantes datos en relación con los hábitos y prácticas de lectura en el país.
} 
fundamental, tanto para la revisión de las propuestas curriculares en la formación universitaria del profesorado, como para futuras intervenciones y mejoras en el tratamiento didáctico de la literatura en la escuela secundaria.

En este sentido, la delimitación del estudio a la lectura de obras literarias obedece no solo a la reconocida importancia de esta para el desarrollo de la competencia lectoliteraria y comunicativa de los jóvenes en la escuela, sino especialmente a la insustituible función de la literatura en la formación individual y social del sujeto, "en todas aquellas dimensiones, como la cognitiva, la imaginaria, la simbólica, la metalingüística o la afectiva entre otras, que no pueden ser desarrolladas por el lenguaje estándar de naturaleza no literaria" (Sánchez Corral, 2003, citado en Ibarra y Ballester, 2016, p. 314). Se trata así de una delimitación que, proyectando el rol mediador de los participantes del estudio, explora las posibilidades de que sus futuros estudiantes "accedan a un discurso modelizador de la experiencia social, utilicen la literatura como instrumento para pensar mundos posibles o para pensar en este de otras formas, para construir la subjetividad y para interpretar la experiencia subjetiva", en el disfrute significativo de "la forma más compleja de articulación discursiva" (Bombini, 1997, citado en Colomer, 2001, p. 18).

El artículo se estructura en tres partes. En la primera de ellas se presenta el diseño de la investigación, con los objetivos y preguntas del estudio, sus bases metodológicas, la identificación de la muestra, la caracterización del instrumento de obtención de datos y el modo de análisis de los mismos. En la segunda parte se presentan los principales resultados de la investigación, con una exposición estadística, análisis y conclusiones particulares de los datos. Finalmente, las conclusiones generales sintetizan los resultados de la investigación en un retrato tipo de las prácticas de lectura literaria declaradas por los estudiantes de PMLC. Junto con ello, se plantean algunas proyecciones para futuros estudios que aborden la relación entre las prácticas de lectura literaria y la formación inicial docente y/o los modos de mediación didáctica en el aula, ya en el contexto del ejercicio profesional docente. 


\section{Diseño de la investigación}

\subsection{Objetivo y preguntas}

El propósito general del estudio fue caracterizar el perfil de lectura literaria de futuros maestros y maestras de PMLC, para la obtención de un retrato lector de mediadores escolares, cuyos hábitos y prácticas de lectura representan una importante variable de influencia en su futura actuación profesional. La pregunta general que orientó la investigación fue: ¿Cómo es el perfil de lectura literaria de los estudiantes egresados de la carrera de PMLC, considerando sus prácticas de lectura personal y académica, en el contexto de sus actividades cotidianas?

Por su parte, dicha pregunta se desglosó en tres preguntas específicas:

- ¿Qué condiciones para la lectura ofrece el entorno familiar y social de estos estudiantes?

- ¿Qué lugar ocupa la lectura en su tiempo libre y actividades cotidianas?

- ¿Qué prácticas personales y académicas de lectura literaria expresan los participantes de la muestra, en términos de cantidad, frecuencia, formas de acceso, autores y libros preferidos, entre otros?

\subsection{Metodología}

Para la caracterización del perfil que se buscaba investigar se realizó un estudio exploratorio y cuantitativo, de base estadística, que posibilitó acercarse a las prácticas de lectura declaradas por los participantes de la muestra. En este proceso, el estudio contempló una primera etapa de documentación acerca de la investigación del comportamiento lector, para luego elaborar y aplicar un cuestionario de recogida de datos a estudiantes egresados de PMLC de Santiago de Chile. Los resultados obtenidos no se manejaron como bases explicativas y/o predictivas, sino que se usaron para caracterizar un perfil de prácticas lectoras declaradas en un momento y lugar específicos, respondiendo al carácter no experimental, con diseño 
transversal del estudio. De este modo, se aseguró la validez interna y fiabilidad del retrato, considerándolo como una representación de la actividad lectora expresada por un importante grupo de futuros profesores y profesoras de Lenguaje y Comunicación de enseñanza secundaria de Chile, pero sin que sus resultados y conclusiones resulten extrapolables a los hábitos y prácticas de lectura literaria de la población total de egresados de PMLC del país.

Lo anterior se complementa, además, con el reconocido hecho de que, en el caso de los estudios en torno a las prácticas lectoras "las declaraciones de los encuestados se sitúan en un espacio híbrido y fluctuante entre las representaciones y los comportamientos reales" (Donnat, 2004, p. 69), lo que exige considerar el impacto del efecto de legitimidad y de sus dos principales dimensiones (Peroni, 2004, s.p.): "una axiológica, que tratándose de lectura tiene una importancia sin equivalencia con otras prácticas culturales (leer es bueno), y una normativa (ciertas lecturas valen más que otras)". De este modo, en la compleja relación entre "las prácticas y las representaciones de las prácticas" (Chartier, 2004, p. 111), el presente estudio se circunscribe a la dimensión declarativa de las mismas, basando sus resultados en lo que los participantes explícitamente han expresado en relación con sus prácticas de lectura literaria.

\subsection{Participantes}

La investigación contempló el trabajo con 80 participantes provenientes de cuatro universidades de Santiago de Chile: dos de ellas públicas y dos privadas, con las cuales se firmó un compromiso de confidencialidad para la participación en la investigación y el análisis y difusión de resultados. Las dos casas de estudios estatales aportaron un total de 42 estudiantes a la muestra, mientras que las dos privadas proporcionaron los 38 restantes. Aunque para la selección se operó con un muestreo por conveniencia, en función de las posibilidades de acceso a los participantes y de su condición de egresados de PMLC de universidades de la Región Metropolitana, el número de participantes del estudio correspondió al 11,4\% de la población de 697 estudiantes que egresaron de esta carrera en 2016 en el país, de acuerdo con la base de datos del Ministerio de 
Educación de Chile, Mineduc (2016). La condición de egresados de los participantes se refiere a estudiantes que, habiendo completado los requisitos de la malla curricular, se encontraban en proceso de titularse como profesores de Lenguaje y Comunicación y ad portas de su ingreso al ejercicio profesional. La elección de la Región Metropolitana responde, por una parte, a la concentración de la oferta académica y matrícula que esta zona presenta en relación con las carreras de PMLC del país y, por otra, a las posibilidades materiales de realización del estudio. La media de edad de los participantes de la investigación fue de 24 años, con una desviación estándar de 2,191, dato que resulta coherente con la situación de egreso de los estudios universitarios para carreras que duran entre ocho y diez semestres. Un 76\% de los participantes correspondió a mujeres (61 estudiantes) y solo un 24\% hombres (19 restantes). Esta distribución por género es equivalente a la población total de titulados de PMLC de 2016 en el país, integrada por un $73,6 \%$ de mujeres y un $26,3 \%$ de hombres.

\subsection{Instrumento de obtención de los datos}

Para la obtención de los datos se utilizó como instrumento un cuestionario individual y estructurado, compuesto por 42 preguntas (abiertas y cerradas) de carácter simple y múltiple. Esta elección se justifica por la reconocida utilidad de este tipo de instrumentos en estudios con enfoques cuantitativos. El proceso de diseño y elaboración del instrumento contempló una etapa preliminar de investigación, en la que se revisaron experiencias de estudio de las prácticas lectoras, tanto del ámbito nacional como internacional, y se contrastaron con un marco teórico de referencia. Como resultado de ello, se llegó a la determinación de las tres dimensiones generales del cuestionario:

- Condiciones del entorno familiar y social para la lectura.

- El uso del tiempo libre y la lectura.

- Prácticas de lectura literaria.

Estas dimensiones resultaron las más relevantes para la construcción de un retrato lector al proveer de una base de análisis que considera inicialmente los elementos más generales de la 
realidad de los estudiantes, para luego explorar las prácticas y usos específicos que estos declaran en relación con la lectura literaria. En este sentido, el modo de operar, tanto en la elaboración de los ítems del instrumento como en el análisis e interpretación de los datos, se hizo sobre la base de las siguientes distinciones:

- Lectura prescriptiva/Lectura por gusto y/o interés personal.

- Lectura de todo tipo de textos/Lectura literaria.

Este modo de plantear el diseño y elaboración del instrumento obedece, por una parte, a la necesidad de situar las prácticas de lectura literaria declaradas por los participantes en un contexto general de actividades y preferencias lectoras (tales como la lectura de otros tipos de textos) y de contrastar las actividades y lecturas "obligatorias" de la formación académica con las que los estudiantes realizan en sus tiempos de ocio.

Luego de elaborar una versión preliminar del instrumento, esta fue sometida a pilotaje con un grupo de ocho estudiantes de tercer año de PMLC de una de las universidades participantes. Una vez generada la versión final del instrumento, se procedió a su aplicación a la muestra en las cuatro universidades. La entrega de instrucciones y condiciones de respuesta fueron las mismas para todos los participantes, sintetizándose el proceso de aplicación en los siguientes pasos:

- breve explicación de los propósitos del estudio,

- firma del consentimiento informado,

- respuesta anónima del cuestionario y

- sellado para su análisis estadístico.

\subsection{Análisis de los datos}

La definición preliminar de las dimensiones de la investigación orientó el proceso de operacionalización de los datos y resultados, el cual se realizó de acuerdo con la lógica tradicional de la estadística descriptiva, atendiendo a la naturaleza cuantitativa o cualitativa de la información y a su tipología. Para facilitar el procesamiento estadístico 
y la tabulación de los datos, la mayor parte de las respuestas del instrumento fue codificada numéricamente. Solo se modificó este procedimiento con la información de las preguntas abiertas, que se sistematizó de acuerdo con la cantidad de coincidencias, obras mayor y menormente citadas y posición numérica de dichas preferencias.

Para procesar los datos se utilizó el programa de análisis estadístico SPSS v.11. Dicho software posibilitó el tratamiento descriptivo y correlacional de la información, con una primera etapa de caracterización del comportamiento independientemente de las dimensiones y criterios, y una segunda de exploración de sus interacciones más relevantes, tales como las referentes a las prácticas de lectura por gusto y/o interés personal y las de la lectura prescriptiva.

\section{Resultados}

Los resultados se presentan mediante descripciones estadísticas, análisis y conclusiones particulares de las dimensiones y criterios definidos para la construcción del retrato lector. De este modo, en una primera etapa se abordan las condiciones del entorno familiar y social para la lectura, con los resultados acerca del nivel educacional de los padres de los participantes, cantidad y tipos de libros en el hogar, cantidad de libros de propiedad de los participantes, frecuencia de lectura de los padres e identificación de las personas del entorno familiar y social que los egresados reconocen como más influyentes en sus hábitos de lectura literaria. En segundo término se presenta el lugar de la lectura entre sus actividades cotidianas, que aporta al retrato datos en torno al lugar que ocupa la lectura entre las actividades diarias de los participantes, los motivos por los que declaran leer y la frecuencia de lectura por gusto y/o interés personal, según diversos tipos de textos. Finalmente, la dimensión sobre las prácticas de lectura literaria presenta los motivos por los que los estudiantes declaran leer literatura, la frecuencia y cantidad de lecturas literarias por gusto y/o interés personal, los aspectos que toman en cuenta al escoger sus lecturas literarias, sus formas de acceso a los textos literarios, los autores y obras que reconocen como importantes en su vida lectora y su autopercepción como lectores de obras literarias. 
El artículo incorpora cuatro gráficos que incluyen información acerca de los tipos de libros en el hogar de los participantes, su percepción acerca de la influencia del entorno familiar y social en sus hábitos de lectura literaria, el lugar de la lectura entre sus actividades cotidianas y los motivos por los que declaran leer literatura.

\subsection{Condiciones del entorno familiar y social para la lectura}

La concepción de la lectura como una práctica social y cultural lleva a poner la mirada en las condiciones materiales e inmateriales del entorno familiar y social en que esta se desarrolla. Este entorno representa un espacio de primera importancia para explorar la relación de vida de los sujetos con la actividad lectora, resultando determinante para, por ejemplo, caracterizar los hábitos y prácticas culturales que dicho contexto ha propiciado.

\subsubsection{Nivel educacional del padre y la madre de los participantes}

En el nivel educacional de los padres de los participantes predominan tres tendencias: un 24,7\% de ellos completó la enseñanza media; un 18,2\% obtuvo además un título de formación técnica y un 18,1\% dejó su enseñanza media sin terminar. A estas tendencias le sigue el $14,3 \%$ de padres con título profesional (entre los cuales existe un $5,2 \%$ con estudios de posgrado); el 10,4\% que dejó la enseñanza básica sin completar; el 7,9\% que sí finalizó dichos estudios; el 5,2\% que abandonó estudios universitarios y un 1,3\% sin escolaridad.

En el nivel educacional de las madres, en tanto, se observan también tres tendencias mayoritarias: un 37,5\% de ellas completó los estudios de enseñanza media; un 18,8\% obtuvo además un título de formación técnica y un 11,3\% dejó la enseñanza media sin terminar. A estas tendencias se agrega un $8,8 \%$ de madres con enseñanza básica completa; igual porcentaje $(8,8 \%)$ que abandonó los estudios de primaria, un 12,6\% que cuenta con un título profesional (con un $6,3 \%$ de ellas con estudios de posgrado) y un 2,5\% que dejó los estudios universitarios sin finalizar. 
En su conjunto, estos datos acerca del nivel educacional de ambos padres de los participantes revelan un bajo porcentaje de progenitores profesionales e importantes índices de deserción escolar, ratificando que la realidad de la mayoría de los estudiantes de la muestra es asimilable a la de los universitarios chilenos de los últimos años, en cuanto a ser primera generación de su familia en acceder a los estudios superiores.

\subsubsection{Cantidad y tipos de libros en el hogar}

En relación con la tenencia de libros impresos, todos los participantes de la encuesta declaran tener libros en sus hogares, con tres grandes grupos destacados: casi un tercio de los encuestados $(32,5 \%)$ expresó contar con 51 a 100 libros, seguido de un $26,3 \%$, que declaró tener entre 201 y 300 ejemplares y un 22,5\% que expresó tener entre 101 y 200 volúmenes. En los rangos de menor cantidad de ejemplares, en tanto, un escaso 2,5\% declaró tener entre 11 y 25 libros; y un $8,8 \%$ lo hizo en relación al rango de 26 a 50 ejemplares. En cuanto al tipo de libro, se observa una mayoritaria existencia de novelas, secundada en un porcentaje mucho menor por libros de poesía y textos escolares, tal como se detalla en el siguiente gráfico:

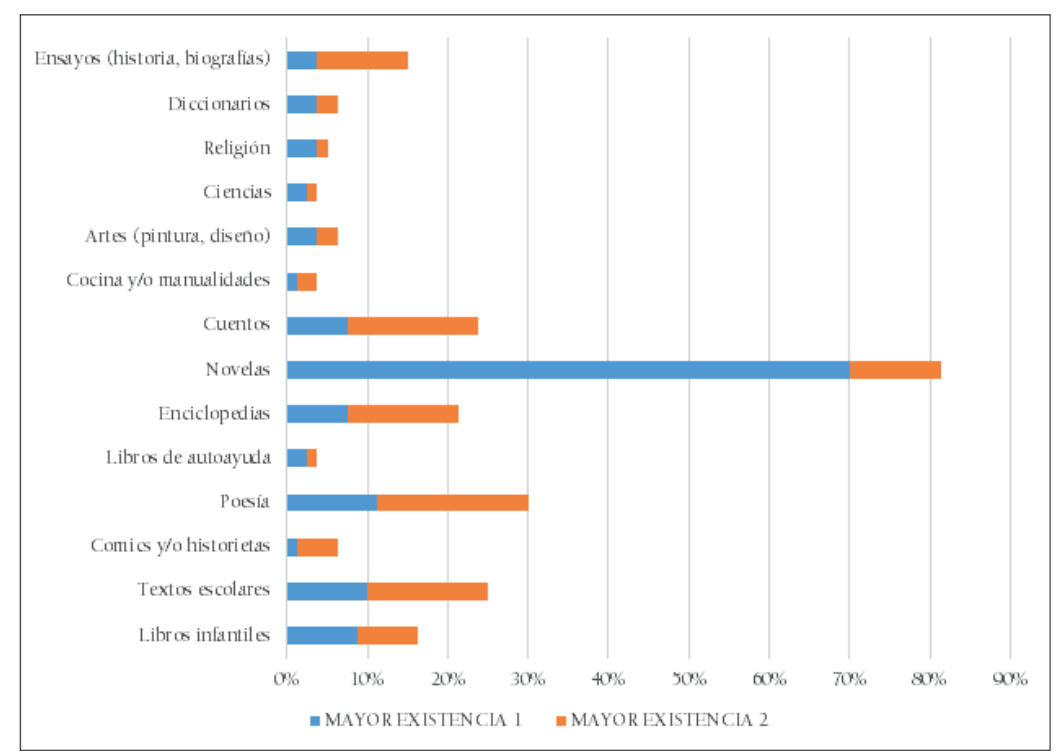

Figura 1. Tipos de libros en el hogar.

Fuente: Elaboración propia. 
A pesar de la escasa diversidad de géneros, estas cifras representan un importante número de participantes en cuyos hogares existe una mayor cantidad de libros que la media nacional, cifrada en 46,9 ejemplares por la encuesta Chile y los libros, de Fundación La Fuente y Adimark (2010). Aunque la tenencia de libros en el hogar resulta, en muchos casos, un factor fundamental para la vinculación temprana con la literatura, se debe considerar, como proponen McQuillan y Au (2001), citados en Manresa (2009, p. 121), que: "El número de libros en el hogar produce un aumento del hábito lector, condicionado por el nivel educativo de los padres y por la habilidad lectora previa de los niños", lo que recuerda que el impacto de la cantidad de libros en el hogar debe medirse en relación con las demás variables del entorno familiar y social, para reconocer, por ejemplo, la procedencia, las formas de uso y los sentidos que dichos materiales de lectura presentan en cada contexto.

\subsubsection{Cantidad de libros de su propiedad}

Atendiendo a lo anterior, resulta significativo comprobar que todos los participantes declaran tener también libros de su propiedad, comprados personalmente o recibidos como regalo. Más aún, las principales tendencias revelan una cantidad considerable de libros personales: la de quienes declaran tener entre 51 y 100 libros de su propiedad (32,5\%), la de quienes expresan tener entre 26 y 50 libros $(18,8 \%)$ y la de quienes declaran entre 101 y 200 volúmenes (16,3\%). La suma de estas tres tendencias representa a casi un $68 \%$ de los estudiantes. Cabe destacar, además, que un 51,2\% de ellos señala que todos o casi todos los libros de su propiedad son textos literarios, mientras que un 38,8\% afirma que más de la mitad de las obras que ha comprado o recibido como regalo son también de literatura. Esta tenencia de libros de literatura se ve también reflejada en la mayoritaria presencia de novelas y, en menor grado, de libros de poesía en el hogar.

Al comparar estas cifras de libros propios con la cantidad de libros en el hogar se observa una correlación directa entre el 32,5\% 
de encuestados que declara tener entre 51 y 100 libros en su hogar y quienes expresan tener una igual cantidad de ejemplares propios. Esto evidencia que, al menos en los hogares donde hay entre $51 \mathrm{y}$ 100 libros (el rango de mayor presencia en la medición de "libros en el hogar" y "libros propios"), los ejemplares son de propiedad directa de los participantes de la muestra.

\subsubsection{Frecuencia de lectura de los padres}

Este rol protagónico de los participantes en la provisión de libros para el hogar se confirma en los datos relacionados con los hábitos de lectura de sus padres. Aunque más de un tercio de los estudiantes $(37,5 \%)$ reconoce al padre y a la madre como lectores frecuentes de distintos tipos de textos ("todos" o "casi todos los días"), el 41,4\% de los participantes proviene de hogares con padres muy poco lectores, que solo leen "algunas veces al mes", "algunas veces al año" o, derechamente, "nunca". Junto con estas tres tendencias de padres poco lectores, existe además un $20 \%$ de padres y madres que solo lee de manera ocasional (solo una o dos veces a la semana, o solo los fines de semana).

Al relacionar esta baja frecuencia de lectura en más de dos tercios de los padres con la propiedad de muchos de los libros del hogar declarada por los participantes, es posible suponer que, en la mayoría de los casos, estos últimos han debido realizar una construcción más bien personal de sus itinerarios de lectura, motivados por actividades escolares y, posteriormente, por los requerimientos de la vida universitaria. Seguramente, esta construcción más o menos tardía de sus itinerarios lectores ha sacrificado, en buena medida, los beneficios de la relación temprana con la lectura y el vínculo personal que ella motiva. Como expresa Cerrillo:

El hábito de la lectura voluntaria suele adquirirse en casa, no en la escuela, siendo una consecuencia de la voluntad de leer, que se ha podido reforzar con la práctica de la lectura en la familia. Lo más eficaz para que un niño lea es, probablemente, que vea leer (2005, p. 137). 
2.1.5. ¿A qué personas del entorno familiar y social reconocen los participantes como más influyentes en sus hábitos de lectura literaria? La ausencia de una relación temprana con la lectura se confirma también en la influencia directa de las personas del entorno familiar y social en los hábitos de lectura literaria de los participantes. Ante la posibilidad de valorar la "mucha", "bastante", "poca" o "nada" influyente relación de los actores de su entorno en sus hábitos lectores, un altísimo 84\% reconoce a sus profesores de universidad como la principal influencia ("mucha" o "bastante"), y un $69 \%$ a sus profesores de enseñanza secundaria. Estas influencias resultan más decisivas que las de la madre, el padre, la pareja, los hermanos y los profesores de primaria, tal como se observa en el siguiente gráfico:

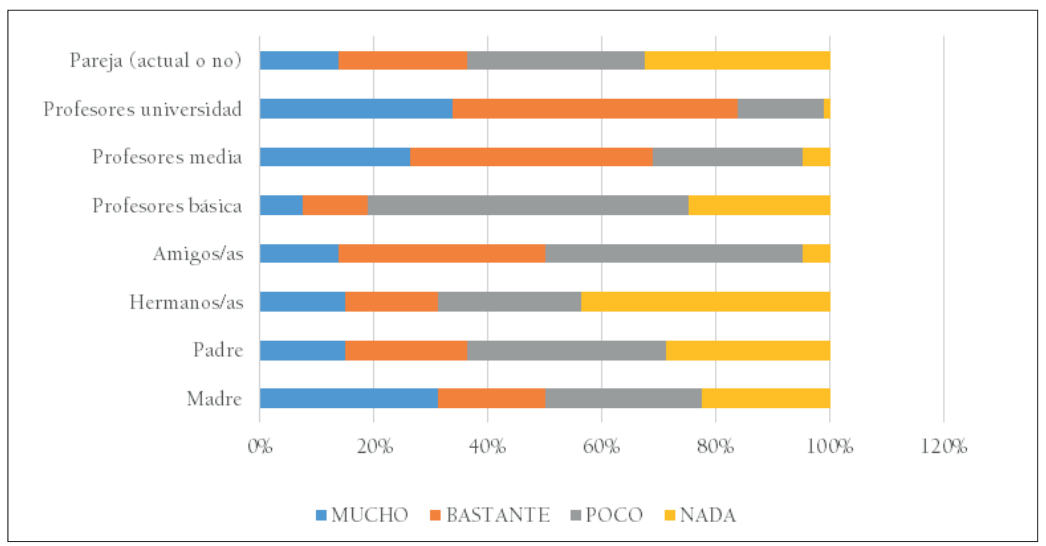

Figura 2. Percepción de los participantes acerca de la influencia de su entorno familiar y social en sus hábitos de lectura literaria.

Fuente: Elaboración propia.

Esta mayoritaria atribución de influencia a sus profesores universitarios y de secundaria en sus hábitos de lectura literaria, junto con la escasa influencia de los profesores de primaria evidencian, al menos, dos cosas importantes para la conformación del retrato lector: el contacto con la lectura literaria ha sido una experiencia más bien reciente en la mayoría de los estudiantes de la muestra y, tan importante como ello, ha estado fundamentalmente asociada a instancias de formación académica, antes que a experiencias vitales y/o de apego temprano a la literatura. 


\subsection{El lugar de la lectura entre sus actividades cotidianas}

Comparar la frecuencia de lectura con las demás actividades cotidianas permitió observar el lugar que la actividad lectora ocupa en la vida diaria de los participantes. El concepto de actividad lectora expresado en este apartado se refiere a la lectura de libros, periódicos, revistas, etc.; sin discriminar aún entre textos literarios y no literarios, como tampoco entre la lectura por gusto y/o interés personal y la que resulta de obligaciones académicas o de otro tipo.

\subsubsection{La lectura entre las actividades cotidianas}

El uso de las redes sociales y la navegación en internet son las actividades que un mayor porcentaje de participantes declara realizar "siempre", con un $61 \%$ y un $56 \%$ de las preferencias respectivamente. No obstante, la lectura (de libros, periódicos y/o revistas) es reconocida también como una actividad realizada "siempre", con un cercano $49 \%$ de preferencias, resultando ser así una práctica muy bien posicionada entre las ocho actividades de mayor presencia en su cotidianidad, tal como se observa en el siguiente gráfico:

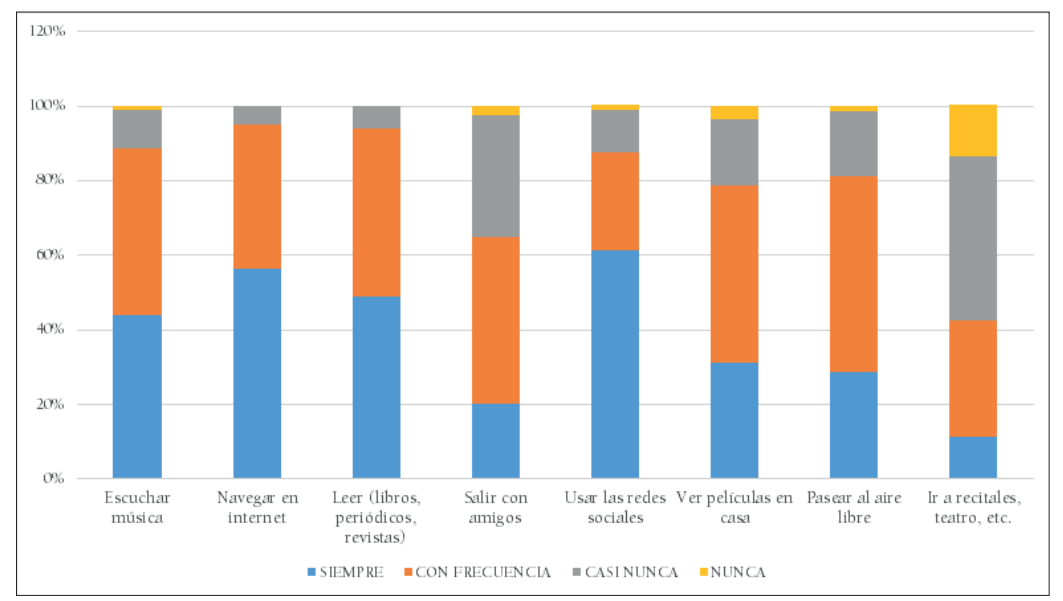

Figura 3. La lectura entre las actividades cotidianas de los participantes.

Fuente: Elaboración propia.

Más aún, al sumar los porcentajes de las actividades realizadas "siempre" y "con frecuencia", la lectura aparece aún mejor situada, superando incluso al uso de las redes sociales y al escuchar música. 
Se debe considerar, sin embargo, que esta presencia cotidiana de la lectura incluye no solo las lecturas personales o recreativas, sino también las prácticas de lectura académica, tales como preparaciones de exámenes y/o evaluaciones.

\subsubsection{Motivos por los que declaran leer}

Una primera delimitación de lo anterior se presenta en los datos referidos a las motivaciones para la lectura. Un 51,2\% de los participantes declara que "el gusto y/o interés personal" es la principal razón por la que lee, indistintamente del tipo de texto. Esta mayoritaria preferencia es secundada, sin embargo, por el $25 \%$ de participantes que afirma que la principal motivación para la lectura es "aprender" y un cercano 21,3\% que declara hacerlo "para cumplir con actividades académicas". La suma de los porcentajes de estas dos últimas motivaciones revela un $46,3 \%$ de encuestados para quienes la lectura de cualquier tipo de textos estaría preferentemente asociada a la formación intelectual y al cumplimiento de actividades académicas.

El equilibrio entre estas dos tendencias tan claras (la de quienes declaran leer por gusto y/o interés personal y la de quienes reconocen motivaciones más bien formativas y prescriptivas para la lectura), complementa los anteriores resultados en torno a las actividades cotidianas y la lectura, mostrando que, aunque los participantes practican la lectura diaria de diversos tipos de textos ello ocurre, en casi igual medida, en el contexto de las actividades académicas de la vida universitaria y en los momentos de ocio. En este último caso, la lectura convive estrechamente con las otras actividades importantes del día: el uso de las redes sociales, la navegación en internet y la escucha de música.

2.2.3. Frecuencia de lectura por gusto y/o interés personal, según tipos de textos

La prensa y revistas de actualidad (deportes, espectáculos, etc.) son los textos que un mayor porcentaje de participantes (43\%) declara leer voluntariamente "a diario". A esta tendencia mayoritaria le sigue la lectura de textos literarios, con un 35\% de indicaciones de lectura diaria. Por otro lado, los textos de religión y autoayuda son los de 
menor frecuencia de lectura libre, con más del 50\% de participantes que declaran no leerlos "nunca"; seguidos de los comics, que son señalados por un $35 \%$ de los encuestados como el tipo de texto que nunca escoge leer.

Esta distribución de los tiempos de lectura libre entre textos informativos y literarios revela una práctica cotidiana de la lectura muy asimilable a los intereses y preferencias de estudiantes de una carrera universitaria como PMLC. El carácter contingente de los textos periodísticos, por una parte, y la experiencia estética con la ficción de las obras literarias, por otra, resultan complementarios con el proceso formativo de los futuros maestros y maestras. No obstante, la lectura libre de textos literarios, que en este apartado se presenta como una actividad relevante en la cotidianeidad de un importante grupo de estudiantes, posee matices, e incluso contradicciones, en la última y principal dimensión del estudio.

\subsection{Prácticas de lectura literaria}

Los resultados de esta sección revelan, inicialmente, los motivos por los que los estudiantes declaran leer literatura y las prácticas de lectura literaria que forman parte de sus actividades de cumplimiento académico, para luego compartir los datos en torno a la lectura literaria que realizan por gusto y/o interés personal. Aunque ambos modos de vinculación con la lectura literaria no resultan antagónicos ni excluyentes - ya que la lectura con propósitos académicos es, también, un aspecto importante en la formación de los lectores literarios-, la identificación de las prácticas de lectura "libre" permite explorar la relación que los estudiantes han desarrollado con la literatura en sus historias de vida lectora.

\subsubsection{Motivos por los que declaran leer literatura}

En este sentido, resulta positivo observar que el 64\% de los participantes declara que la principal razón por la que lee literatura es "el gusto y/o interés personal", mientras que el 19\% manifiesta que la principal razón por la que lo hace es "para cumplir con actividades académicas" y el 15\%, "para aprender". El siguiente gráfico presenta también los motivos secundarios, terciarios y menos influyentes 
según los estudiantes, como motivación personal para la lectura literaria:

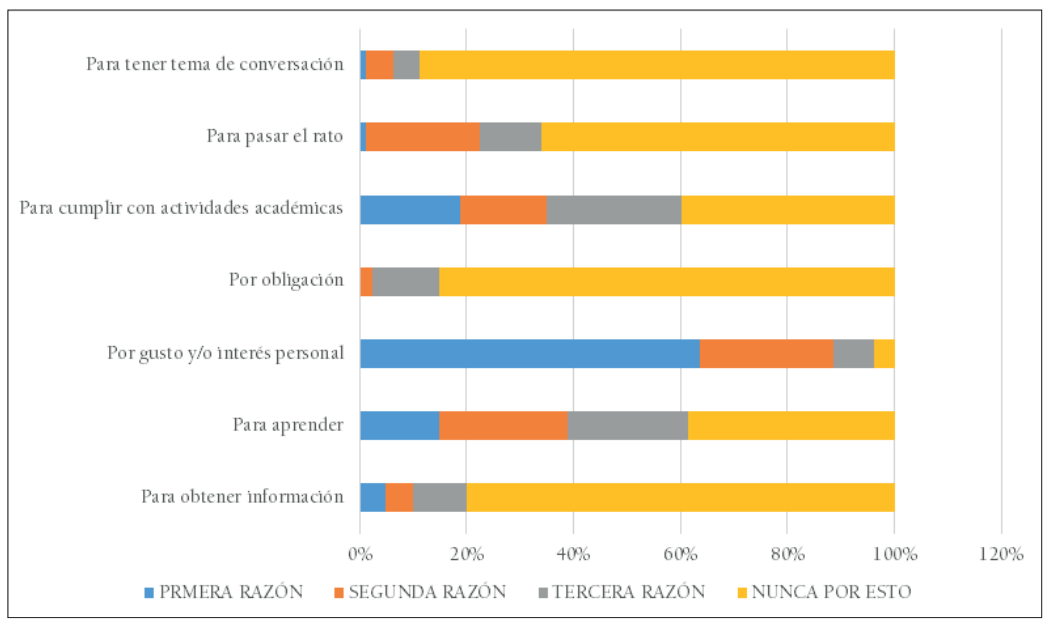

Figura 4. Motivos por lo que los participantes declaran leer literatura.

Fuente: Elaboración propia.

Dos aspectos resultan importantes de destacar en este punto. El primero es la diferencia de género que se presenta en las motivaciones para la lectura. Mientras el porcentaje de hombres que asegura leer literatura "para cumplir con requisitos académicos" corresponde a un $42 \%$ y el de quienes declaran hacerlo "para aprender" a un $21 \%$, estas razones se presentan solo en unas cifras cercanas a la mitad de las mujeres (un 21\% y 13\%, respectivamente). Estas diferencias expresan una motivación más académica e instrumental para la lectura literaria en los hombres y una relación más personal y libre con este tipo de lectura de las participantes mujeres. El segundo aspecto es que, a pesar de que una parte mayoritaria de la muestra declara una libre motivación para la lectura de textos literarios, la suma de quienes expresan que la principal razón por la que lee literatura es "para cumplir con actividades académicas" y la de quienes declaran que su principal motivación es "aprender" alcanza a un 34\%; esto es, más de un tercio de futuros profesores para quienes la motivación para la lectura literaria surge de las circunstancias formativas y curriculares de su vida universitaria. 
2.3.2. Frecuencia y cantidad de lecturas literarias por gusto y/o interés personal

La predominante motivación del "gusto y/o interés personal" para la lectura expresada en el punto anterior contrasta fuertemente con la cantidad de libros leídos en los últimos doce meses. Ante la consulta, casi un tercio de los estudiantes (30\%) reconoce la lectura de solo entre tres y cinco ejemplares por gusto y/o interés personal en dicho periodo. Si a este bajo porcentaje se agrega el de quienes declaran haber leído solo uno o dos libros (13\%), se registra un 43\% total de participantes que en el último año recuerda haber leído solo entre uno y cinco libros de literatura por interés personal y/o recreativo.

En este punto también se presentan importantes diferencias de género. Aunque en los rangos de lectura media existe un ligero porcentaje a favor de los hombres, con un 16\% de lectores que declara haber leído entre 11 y 15 libros en el último año y un $11 \%$ que expresa haber leído entre 16 a 20 ejemplares; el 16\% de lectores fuertes (que leyó 21 libros y más en los últimos doce meses) corresponde solo a mujeres, mientras que el 43\% de lectores débiles (que leyó solo entre uno y cinco libros en el último año) está compuesto por casi igual porcentaje de hombres y mujeres.

Estas cifras, que en general muestran una baja frecuencia y cantidad de lectura no prescriptiva en la mayoría de los participantes, deben considerarse a la luz de la dificultad personal que implica recordar, con precisión, una cantidad de libros leídos. Se trata de una operación que "exige a la persona encuestada la construcción de una variable 'objetiva' que ella desconoce. ¿Quién hace un cálculo de este tipo en la vida cotidiana?" (Donnat, 2004, p. 69).

No obstante lo anterior, estas cifras podrían estar sugiriendo también una interesante evidencia acerca del dato cualitativo del "gusto y/o interés personal" como principal motivación para la lectura declarado por la mayoría de los estudiantes: una cosa es la libre motivación que reconocen como predominante en su relación con la lectura literaria, pero otra es que dicha motivación los lleve efectivamente a leer. 
2.3.3. ¿Qué aspectos toman en cuenta al escoger sus lecturas literarias? Ante la consulta por los factores de influencia para la elección de sus lecturas literarias, "la temática de la obra" y "el género" resultan ser los más decisivos con el $42,5 \%$ y el $23,8 \%$ de las preferencias respectivamente, mientras que un $13,8 \%$ declara su "conocimiento personal del autor" y solo un 11,3\% la "recomendación de un profesor". En tanto, en los aspectos poco o nada influyentes destacan "la difusión de la obra en los medios", que solo recibe un 1,3\% de las preferencias y, especialmente, "la recomendación de los familiares" y "las imágenes de la portada", que no fueron señalados por ninguno de los participantes como un factor de influencia para la elección de sus lecturas literarias.

Una pregunta que surge de estos datos es ¿cómo se explica que, mayoritariamente, los estudiantes reconozcan a sus profesores (principalmente universitarios, pero también de secundaria) como las personas "más influyentes en sus hábitos de lectura literaria" y luego sea tan bajo el porcentaje de ellos que declare influyente "la recomendación de un profesor" para la lectura recreativa de textos literarios? Esta contradicción parece confirmar la influencia más bien prescriptiva de los profesores, quienes seguramente han puesto a los participantes de la muestra en contacto con las obras y autores en el contexto de actividades escolares y académicas, pero sin necesariamente incidir —al menos no de manera decisiva- en sus prácticas personales de lectura.

\subsubsection{Formas de acceso a los textos literarios}

Las "descargas de internet" y "la colección de libros del hogar" resultan ser las vías de acceso más frecuentes a la literatura que los participantes leen por gusto y/o interés personal. Mientras internet es considerado por un 41,3\% de ellos como el modo de acceso que "siempre" usa para leer literatura y por un 23,8\% como el que usa "con frecuencia", la colección de libros del hogar resulta tan importante como este, al ser identificado por un $40 \%$ como la forma por la que "siempre" accede a las obras literarias y por un 41,3\% como la vía de acceso que ocupa "con frecuencia". En tanto, la "compra personal de libros" (nuevos o usados) es la tercera forma de acceso más relevante 
a los textos literarios, con un 23,8\% de participantes que declaran practicarla "siempre" y un importante $47,5 \%$ que reconoce hacerlo "con frecuencia".

En el lado opuesto de las preferencias, los préstamos de las bibliotecas públicas y de la universidad se revelan como las alternativas menos usadas por los estudiantes para leer literatura de manera recreativa. En la suma de los indicadores negativos del ítem, el 67,5\% de los participantes reconoce que "nunca" y "casi nunca" usa las bibliotecas públicas, mientras que el 58,8\% afirma lo mismo en cuanto a la biblioteca de la universidad para acceder a la lectura de textos literarios. Por su parte, los préstamos de libros de los amigos y/o familiares aparecen como una opción muy equilibrada estadísticamente, con un 43,8\% de los participantes que declara que "casi nunca" accede de esa manera a los libros y un 40\% de ellos lo hace por esa vía "con frecuencia".

En el panorama general, estos datos evidencian que la lectura recreativa de textos literarios está circunscrita a un ámbito más bien individual en la vida de los participantes, al acceder a dichas lecturas desde las vías más inmediatas (como internet y los libros en casa), prescindiendo de la rica exploración de las bibliotecas. En lo positivo, no obstante, los datos de acceso confirman que más de dos tercios de los estudiantes tiene hábitos de compra de libros de literatura, estadística que además se correlaciona muy directamente con el 68\% de los estudiantes que posee importantes cantidades de libros de su propiedad.

\subsubsection{Autores favoritos}

Ante la opción de nombrar hasta cinco autores favoritos, un 22,5\% de los participantes identificó cinco autores y otro 22,5\% un solo autor, reiterándose en ambas tendencias el nombre de tres escritores latinoamericanos: Gabriel García Márquez, Julio Cortázar y Roberto Bolaño, en dicho orden de referencias. Sin embargo, junto con estas dos tendencias, un no menor 18,8\% de los participantes tomó la opción de dejar la pregunta en blanco, expresando no tener o no recordar autores favoritos de literatura. 
106 LA LECTURA LITERARIA AL EGRESO DE LA FORMACIÓN INICIAL DOCENTE. UN RETRATO DE LAS PRÁCTICAS LECTORAS DECLARADAS POR ESTUDIANTES DE PEDAGOGÍA EN EDUCACIÓN SECUNDARIA EN LENGUAJE Y COMUNICACIÓN - E. Asfura, N. Real

En la referencia general de autores (con dos, tres o cuatro menciones por participante) en tanto, se reitera la tendencia de la narrativa latinoamericana: Juan Rulfo, Alberto Fuguet, María Luisa Bombal, José Donoso, Ernesto Sábato, junto a la identificación más esporádica de poetas como Pablo Neruda y Mario Benedetti y autores europeos como Hermann Hesse, Víctor Hugo y Franz Kafka.

El elevado porcentaje de estudiantes que no reconoce autores favoritos o que menciona uno solo — cuya suma alcanza a un 41,3\% de la muestra - resulta muy asimilable con otros datos del estudio, como el del 42,5\% de participantes que presenta una escasa cantidad de lecturas recreativas de textos literarios en los últimos 12 meses o la de un igual porcentaje $(42,5 \%)$ que declara escoger preferentemente sus lecturas por la temática de la obra, antes que por el seguimiento de determinadas poéticas de autor.

\subsubsection{Obras importantes en su vida lectora}

La baja referencia a autores favoritos en el punto anterior contrasta con la mayoritaria declaración acerca de las obras literarias importantes en su vida lectora. Solo un $5 \%$ de los participantes dejó este ítem sin responder, mientras que un 66,2\% reconoció tener al menos cuatro o cinco obras favoritas, presentándose como referencia mayoritaria el libro Cien años de soledad, de Gabriel García Márquez, con un 11,5\% de las coincidencias en primer lugar y un 5\% en el segundo puesto.

Como es previsible, en el caso de las obras se presenta una mayor disparidad de títulos que en el de los autores favoritos. No obstante, se mantiene la preferencia mayoritaria por la narrativa, aunque ya no tan estrechamente acotada a los autores latinoamericanos. Esto resulta evidente al considerar las obras favoritas sobre las que se presenta un mayor número de coincidencias, sin importar la posición en que son referenciadas. Títulos como La insoportable levedad del ser (Milan Kundera), Don Quijote de la Mancha (Miguel de Cervantes), El Principito (Antoine de Saint Exupéry), Crimen y castigo (Fiódor Dostoyevski), Los miserables (Víctor Hugo) y Rayuela (Julio Cortázar) comparten lugar en las preferencias con best sellers como Harry Potter (Joanne K. Rowling) y Los juegos del hambre (Suzanne Collins). 
En el conjunto, esta referencia de obras importantes en la vida de los participantes remite a unos itinerarios lectores centrados casi exclusivamente en el género narrativo y a una relación más o menos abierta entre el canon de la narrativa clásica y contemporánea y los best sellers de reciente publicación. Considerando el tipo de obras, es posible interpretar, además, que su lectura data de un periodo más o menos reciente y, en muchos casos (como el de Don Quijote de la Mancha y Los Miserables), que puede resultar más bien atribuible al contexto de la formación disciplinaria de la profesión docente.

\subsubsection{Autopercepción como lectores de literatura}

Finalmente, ante la consulta por su autopercepción como lectores de literatura el 70\% de los participantes de la muestra declara sentirse "un lector promedio"; es decir, que "lee permanentemente, aunque a veces por gusto y otras por obligación"; mientras que solo un $25 \%$ de los encuestados reconoce ser "un gran lector", declarando "leer abundantemente, de forma constante y disfrutando la mayoría de las lecturas". El 5\% restante de los participantes se percibe como "un lector ocasional", reconociendo "leer solo de vez en cuando y la mayoría de veces por obligación”.

Estos datos de autopercepción de la identidad lectora resultan muy coherentes con las prácticas de lectura declaradas por un importante grupo de la muestra. De manera específica, la autopercepción del 25\% de los participantes como "un gran lector" correlaciona de manera directa con el 26,9\% de estudiantes que declara una lectura de 15 o más libros por gusto y/o interés personal en los últimos 12 meses. Y también se halla estadísticamente muy cerca del 22,5\% de participantes que menciona el máximo de cinco autores favoritos en el ítem correspondiente y el 26,3\% que declara el mayor volumen de ejemplares (entre 200 a 500) en la estadística sobre la tenencia de libros en el hogar.

Estos datos permiten concluir que al menos un cuarto de los estudiantes (20 participantes) posee una autopercepción lectora que se ajusta a sus prácticas de lectura literaria. Y que dichas prácticas expresan un vínculo temprano y significativo con la lectura, en unas condiciones claramente distintas de las del grueso de los participantes. 
108 LA LECTURA LITERARIA AL EGRESO DE LA FORMACIÓN INICIAL DOCENTE. UN RETRATO DE LAS PRÁCTICAS LECTORAS DECLARADAS POR ESTUDIANTES DE PEDAGOGÍA EN EDUCACIÓN SECUNDARIA EN LENGUAJE Y COMUNICACIÓN - E. Asfura, N. Real

\section{Conclusiones}

De todo lo anterior se deriva un retrato tipo de las prácticas de lectura literaria declaradas por los egresados de la carrera de PMLC de las cuatro universidades de la Región Metropolitana, de acuerdo con las dimensiones definidas para su caracterización: condiciones del entorno familiar y social para la lectura, el lugar de la lectura entre sus actividades cotidianas y prácticas de lectura literaria. El retrato se concreta, así, en lo siguiente.

El contexto sociocultural de los participantes corresponde, en general, al de los estudiantes universitarios chilenos de la última década, en cuanto a ser la primera persona de su familia en acceder a la educación superior. Esto implica que algunos factores decisivos para el contacto temprano y significativo con la literatura, tales como la escolaridad y hábitos de lectura de sus padres, se proyectan como poco favorables en sus historias de vida lectora. Coherentemente con esto, las principales influencias en los hábitos y prácticas de lectura literaria declaradas por los participantes no provienen predominantemente del hogar y la familia, sino de la escuela secundaria y de la educación superior. Sus profesores universitarios y, en menor medida, sus maestros de secundaria, han sido los encargados de "hacer las presentaciones" (Colomer, 2002, citada en Munita, 2014) entre los participantes y los libros. Esta influencia de sus profesores demuestra no solo un contacto más o menos reciente con la lectura de textos literarios, sino especialmente un vínculo generado en el contexto de actividades académicas y escolares. Y como se sabe, resulta muy distinto adquirir un apego temprano por la literatura en un entorno que lo ha propiciado, a "tener que leer" determinadas obras y autores como parte de un proceso de formación académica y profesional. Ante ello, resulta previsible que, en la mayoría de los casos, esta situación genere un importante sesgo instrumental en las lecturas y modos de experimentar con la literatura.

Este sesgo instrumental no significa, sin embargo, que los estudiantes de la muestra no se hayan formado como lectores de literatura. De hecho, un cuarto de ellos expresa unos hábitos y prácticas lectoras muy consolidados en cuanto a frecuencia, cantidad, 
motivaciones y tipos de lecturas. De igual manera, en la totalidad de los hogares de la muestra se observa la existencia de libros (aunque con poca diversidad de géneros y tipos de obras) y la propiedad personal de libros de literatura, comprados o recibidos como regalo por los participantes. Pero en general, estas condiciones favorables para el cultivo del hábito lector parecen ser resultado de la acción de los estudiantes sobre su entorno y no al revés. Y dicha acción ha estado motivada, mayoritariamente, por las demandas escolares y académicas.

Lo anterior resulta muy claro en la dimensión sobre el lugar que ocupa la lectura en las actividades cotidianas de los participantes. Aunque el uso de las redes sociales y la navegación en internet son las actividades prioritarias de su día a día, el retrato muestra también una lectura permanente de textos impresos. Las motivaciones para dicha actividad se hallan, sin embargo, divididas entre un grupo para el cual el acto de leer se asocia fundamentalmente con la formación intelectual y con el cumplimiento de actividades curriculares y otro, casi de igual proporción, para el que la principal motivación lectora es, propiamente, el gusto y/o interés personal. En ese sentido, sus prácticas de lectura resultan muy homogéneas, con un importante predominio de la lectura recreativa de prensa (especialmente deportiva), seguida por la lectura prescriptiva y libre de literatura y la lectura estrictamente formativa de los textos académicos.

En estrecha relación con lo anterior, la dimensión sobre las prácticas de lectura literaria de los participantes revela unos hábitos y prácticas lectoras influidos por una importante tensión entre la experiencia personal y las exigencias de la vida universitaria. Dicha tensión se asocia con los esfuerzos que los estudiantes han hecho para "aprender a disfrutar" las obras en un contexto de formación académica y profesional, pero privándose en buena medida del vínculo más libre que el contacto temprano con la literatura posibilita. Esta tensión entre la lectura fruitiva y la impuesta por la vida universitaria resulta muy clara, por ejemplo, en las motivaciones libres y personales que mayoritariamente declaran como prioritarias para la lectura de textos literarios y la baja frecuencia y cantidad de lectura que muchos de ellos reconocen. 
A este aprendizaje tardío del gusto se debe, tal vez, el que la mayoría de estos estudiantes presente un repertorio más bien homogéneo de lecturas, en términos de preferencias y títulos comunes. Dicho repertorio está casi estrictamente circunscrito al género de la narrativa, con una fuerte inclinación por las novelas latinoamericanas. Aunque dicha homogeneidad y coincidencias pudieran interpretarse como las naturales similitudes de gustos en un mismo grupo etario, resulta significativo que las preferencias se diversifiquen en el caso de quienes declaran una mayor cantidad y frecuencia de lecturas. Esta mayor diversificación de gustos, reconocible en el $25 \%$ de participantes que se ha revelado como "lectores fuertes", demuestra que el vínculo personal con la literatura se traduce en una búsqueda más rica y diversa de lecturas. Pero en general, el grueso de los egresados se perfila como lectores poco dispuestos a la exploración. Sus decisiones de lectura se orientan preferentemente por las temáticas de las obras narrativas, antes que por el seguimiento de determinadas poéticas de autor y/o la valoración de las posibilidades estéticas del lenguaje. Esta escasa tendencia a la exploración en la lectura personal de literatura se da, sin embargo, en el marco de una mayoritaria incorporación de soportes digitales como nuevas formas de acceso a la lectura. Siguiendo una tendencia global (y muy asimilable con la cantidad de tiempo diario que destinan a la navegación en internet), el número de participantes que declara acceder siempre a la literatura recreativa a través de la red resulta, incluso, levemente superior a quienes expresan hacerlo mediante libros impresos, aunque al considerar el conjunto de lecturas por gusto y/o interés personal y lecturas prescriptivas, los libros impresos continúan siendo el formato más frecuentado.

De todo lo dicho se proyecta una figura de docente para el que la literatura resulta más bien un objeto de aprendizaje escolar y académico, antes que una experiencia autónoma y vital, instaurada como una práctica legítima desde sus espacios de ocio y recreación. Aunque el carácter de dicha formación lectora plantea interrogantes acerca del rol de este grupo de nuevos profesores de PMLC como mediadores escolares de lectura literaria y sus posibilidades para, por ejemplo, innovar en dicho rol, esta construcción instrumental de sus itinerarios de lectura no debe considerarse, por sí sola, una variable 
de valoración del conjunto de su actuación profesional. Como se ha dicho, este retrato constituye un acercamiento exploratorio a las prácticas de lectura literaria en el momento del egreso de la formación inicial docente de estudiantes de PMLC de Santiago de Chile. Por consiguiente, sus datos, análisis y conclusiones señalan tendencias, que no resultan directamente extrapolables a las prácticas de lectura de todos los egresados de PMLC del país. Esta limitación no solo se relaciona con la cantidad de estudiantes participantes de la muestra y la zona geográfica a la que se circunscribe el estudio, sino también con el carácter predominantemente estadístico del mismo. Aun así, la investigación aporta información de primer orden acerca del perfil del egresado de PMLC y, por tanto, del futuro docente de enseñanza secundaria, proyectando interesantes líneas de continuidad que, por una parte, sugieren la necesidad de realizar estudios cualitativos y etnográficos en torno a las experiencias de vida lectora del profesorado (en formación o en ejercicio) y, por otra, revelan la importancia de vincular dichas experiencias con la actuación profesional docente y las formas de mediación en el aula, desde los desafíos que la noción de sujeto lector didáctico (Munita, 2018) representa para el fomento de la lectura en el contexto de la escuela básica y secundaria chilena.

\section{Referencias}

Applegate, A. J. \& Applegate, M. D. (2004). The Peter effect: Reading habits and attitudes of preservice teachers. The Reading Teacher, 57(6), 554563.

Chartier, A. M. (2004). La memoria y el olvido, o cómo leen los jóvenes profesores. En B. Lahire (Coord.), Sociología de la lectura. Del consumo cultural a las formas de la experiencia literaria (pp. 109-137.) Barcelona: Gedisa.

Cerrillo, P. (2005). Los nuevos lectores: la formación del lector literario. En M. C. Utanda, P. Cerrillo, y J. García (Coords.), Literatura infantil y educación literaria (pp. 135-157). La Mancha: Ediciones de la Universidad de Castilla.

Colomer, T. (2001). La enseñanza de la literatura como construcción de sentido. Lectura y Vida Revista Latinoamericana de Lectura, 22(1), 6-23.

Colomer, T. (2005). Andar entre libros. México D.F.: Fondo de Cultura Económica. 
112 LA LECTURA LITERARIA AL EGRESO DE LA FORMACIÓN INICIAL DOCENTE. UN RETRATO DE LAS PRÁCTICAS LECTORAS DECLARADAS POR ESTUDIANTES DE PEDAGOGÍA EN EDUCACIÓN SECUNDARIA EN LENGUAJE Y COMUNICACIÓN - E. Asfura, N. Real

Donnat, O. (2004). Encuestas sobre comportamiento de lectura. Cuestiones de método. En B. Lahire (Coord.), Sociología de la lectura. Del consumo cultural a las formas de la experiencia literaria (pp. 59-84). Barcelona: Gedisa.

Fundación La Fuente y Adimark G.F.K. (2010). Chile y los libros. Recuperado de http://www.fundacionlafuente.cl/wp-content/uploads/2010/11/ Chile-y-los-libros-2010_FINAL-liviano.pdf

Ibarra, N. y Ballester, J. (2016). La literatura en la formación universitaria desde el Espacio Europeo de Educación Superior. Alpha (Osorno), 43, 303-317. https://doi.org/10.4067/s0718-22012016000200022

García-Huidobro, J. E. (2009). El papel de la lectura en la formación de profesores. En Fundación Santillana (Ed.), La lectura en la sociedad de la información. XXIII Semana Monográfica de la Educación (pp. 159166). Madrid: Autor.

Gilardoni, C. (2006). Universitarios y lectura: análisis cuali-cuantitativo del uso, accesibilidad y valoración de los libros. Revista Calidad en la Educación, 25, 215-239. https://doi.org/10.31619/caledu.n25.260

Granado, C. y Puig, M. (2014). ¿Qué leen los futuros maestros y maestras? Un estudio del docente como sujeto lector a través de los títulos de libros que evocan. Ocnos. Revista de Estudios Sobre Lectura, 11, 93-112. Recuperado de http://www.revista.uclm.es/index.php/ocnos/article/ view/441

Gupta, R. (2004). Old habits die hard: Literacy practices of preservice teachers. Journal of Education for Teaching, 30(1), 67-78. https://doi.or g/10.1080/0260747032000162325

Jauss, H. R. (1992). Experiencia estética y hermenéutica. Madrid: Taurus.

Jiménez, M. y Lagos, F. (2011). Nueva geografía de la educación superior y de los estudiantes: una cartografía del sistema chileno, su actual alumnado y sus principales tendencias. Santiago de Chile: Ediciones Universidad San Sebastián.

Leyton, D., Vásquez, A., y Fuenzalida, V. (2012). La experiencia de estudiantes de contextos vulnerables en diferentes Instituciones de Educación Superior Universitaria (IESU): resultados de investigación. Revista Calidad en la Educación, 37, 61-97. https://doi.org/10.31619/caledu. n37.87

Lluch, G. (2010). Las nuevas lecturas deslocalizadas de la escuela. En G. Lluch, E. Gil Calvo, J. M. Barbero, R. Morduchowicz, G. A. Arellano, y P. Cerrillo (Eds.), Las lecturas de los jóvenes. Un nuevo lector para un nuevo siglo (pp. 105-128). Barcelona: Editorial Anthropos. 
Manresa, M. (2009). Els hàbits lectors del adolescents. Efectes de les actuacions escolars en les pràctiques de lectura (Tesis doctoral inédita). Universitat Autònoma de Barcelona, Barcelona, España.

Mayol, A. (2014). La lectura como práctica social: condiciones de fortalecimiento y debilitamiento de la lectura en Chile. Santiago de Chile: Observatorio del Libro y la Lectura, Consejo de la Cultura y las Artes.

Ministerio de Educación de Chile, Mineduc. (2016). Estadísticas por carrera. Recuperado de http://www.mifuturo.cl/index.php/futuro-laboral/ buscador-por-carrera?tecnico=false $\$ \mathrm{cmbareas}=7 \& \mathrm{cmbinstituciones=3}$

Munita, F. (2013). Creencias y saberes de futuros maestros (lectores y no lectores) en torno a la educación literaria. Ocnos. Revista de Estudios Sobre Lectura, 9, 69-87. https://doi.org/10.18239/ocnos_2013.09.04

Munita, F. (2014). El mediador escolar de lectura literaria. Un estudio del espacio de encuentro entre prácticas didácticas, sistemas de creencia y trayectorias personales de lectura (Tesis doctoral inédita). Universitat Autònoma de Barcelona, Barcelona, España.

Munita, F. (2018). El sujeto lector didáctico: "lectores que enseñan y profesores que leen". Álabe Revista de Investigación sobre Lectura y Escritura, 9(17), 1-19. https://doi.org/10.15645/alabe2018.17.2

Pascual Díez, J. (2007). Lecturas y factores que condicionan la maduración lectora. Implicaciones educativas. En P. Cerrillo, C. Cañamares, y C. Sánchez (Coords.), Literatura infantil: nuevas lecturas, nuevos lectores (pp. 421-428). Cuenca: Ediciones Universidad de Castilla La Mancha.

Peroni, M. (noviembre, 2004). La lectura como práctica social. Los equívocos de una evidencia. Conferencia magistral pronunciada en el II Encuentro de Promotores de la Lectura. XVIII Feria Internacional del Libro de Guadalajara, Guadalajara, México.

Rosenblatt, L. (2002). La literatura como exploración. México D.F.: Fondo de Cultura Económica.

Sánchez Corral, L. (2008). De la competencia literaria al proceso educativo: actividades y recursos. En A. Mendoza Fillola (Comp.), Didáctica de la lengua y la literatura (pp. 319-348). Madrid: Pearson Educación. 